\title{
A REPRODUCING KERNEL FORMULATION FOR MODELING SHOCKS
}

\author{
Jason Roth $^{1}$, JS Chen ${ }^{2}$, Tom Slawson ${ }^{1}$, Kent Danielson ${ }^{1}$ \\ ${ }^{1}$ U.S. Army Engineer Research and Development Center, Vicksburg, MS \\ (michael.j.roth@usace.army.mil) \\ ${ }^{2}$ University of California, Los Angeles, Department of Civil and Environmental Engineering
}

\begin{abstract}
A reproducing kernel particle method formulation for modeling shocks formed by nonlinear hyperbolic partial differential equations is developed using a flux-based velocity correction technique. The technique is coupled with a spectral decomposition shock detection algorithm to isolate corrections to the jump location. For this class of model problems, the technique is shown to accurately capture the physically correct solution and minimize oscillatory error due to Gibbs phenomenon. The approach provides a basis for further investigation on the extension to the equation of motion and shock-forming solid dynamics problems.
\end{abstract}

Keywords: reproducing kernel, shock physics, spectral decomposition, Gibbs phenomenon

\section{INTRODUCTION}

Strong dynamic events such as projectile penetration and blast effects generate extreme loads in solids, which are typically accompanied by internal shock propagation, high strain rates, large deformations, and material fragmentation. Meshfree methods are well suited to model these phenomena, where evolving contact surfaces, large deformation, and material separation can be accurately captured in the meshfree framework $[2,8]$. The reflection of strong internal shock waves at material interfaces plays a particularly important role in penetration and blast effects. With certain changes in material impedance, initially compressive shock waves can generate strong tensile wave reflections that result in dynamic tensile failure. Internal wave reflections in heterogeneous materials (such as concrete) lead to microcrack growth and reinforcement debonding, while free-surface reflections can generate dynamic spall. Both cases contribute to the evolution of material damage at the macroscale and result in global failure of structural systems.

To model strong shock effects, a numerical formulation must accurately capture the shock wave formation, propagation, and complex interactions. This requires that the key shock physics be embedded in the formulation. According to Leveque [1], the essential ingredients of a numerical method to model shocks are 
1. Consistency and stability to guarantee solution convergence,

2. Conservation of the conserved quantities,

3. Satisfaction of the Rankine-Hugoniot jump condition,

4. Satisfaction of the entropy condition at a shock,

5. Maintenance of high-order accuracy in regions of smooth solution, and

6. Sharp shock-front resolution without oscillation (Gibbs phenomenon) or excessive dissipation.

The first two requirements must be satisfied by a valid numerical method and they can be met by the Reproducing Kernel Particle Method (RKPM). The third and fourth requirements are addressed by inclusion of the appropriate shock physics, and the last two requirements are generally related to construction of the numerical approximation.

An RKPM-based formulation for satisfaction of the key physics associated with shock formation and propagation is discussed in this paper. A method to maintain high-order accuracy in smooth regions and accurately capture the fine scale shock-front motion is presented. A numerical example using the inviscid Burgers' equation is given.

\section{REPRODUCING KERNEL PARTICLE METHOD}

The Reproducing Kernel Particle Method [2,3] is a Galerkin meshfree method that is typically formulated in a Lagrangian framework. The solution of governing partial differential equations is approximated using a meshfree domain discretization, in which the nodes interact through nonconforming kernels of compact support. The nonconforming characteristic of the approximation avoids problems with structured-mesh dependency, making the method ideal for large deformation problems, particularly in the presence of evolving contact surfaces and material fragmentation. A reproducing kernel (RK) discretization is shown in Figure 1.

In the following, the general $d$-dimensional notation is used, where $x^{\alpha}=x_{1}^{\alpha_{1}} \cdots x_{d}^{\alpha_{d}}$ and $|\alpha|=\sum_{i=1}^{d} \alpha_{i}$. In the reproducing kernel approximation of degree $n$, the approximation of an unknown field variable, denoted by $\boldsymbol{u}^{h}(\boldsymbol{x})$, is

$$
\boldsymbol{u}^{h}(\boldsymbol{x})=\sum_{I=1}^{n} C\left(\boldsymbol{x} ; \boldsymbol{x}-\boldsymbol{x}_{I}\right) \Phi_{a}\left(\boldsymbol{x}-\boldsymbol{x}_{I}\right) \boldsymbol{d}_{I} \equiv \sum_{I} \Psi_{I}(\boldsymbol{x}) \boldsymbol{d}_{I}
$$

where $\Psi_{I}(\boldsymbol{x})$ is the RK shape function, $\Phi_{a}\left(\boldsymbol{x}-\boldsymbol{x}_{I}\right)$ is a scalar-valued kernel function with compact support of size $a, \boldsymbol{d}_{I}$ is the RK approximation coefficients for displacement, and $C\left(\boldsymbol{x} ; \boldsymbol{x}-\boldsymbol{x}_{I}\right)$ is the correction function expressed as

$$
C\left(\boldsymbol{x} ; \boldsymbol{x}-\boldsymbol{x}_{I}\right)=\boldsymbol{H}^{T}\left(\boldsymbol{x}-\boldsymbol{x}_{I}\right) \boldsymbol{b}(\boldsymbol{x})
$$

where $\boldsymbol{H}^{T}\left(\boldsymbol{x}-\boldsymbol{x}_{I}\right)=\left\{\left(\boldsymbol{x}-\boldsymbol{x}_{I}\right)^{\alpha}\right\}_{|\alpha| \leq n}$ is a vector of monomial basis functions of degree $n$, and

$\boldsymbol{b}^{T}(\boldsymbol{x})=\left\{b_{\alpha}(\boldsymbol{x})\right\}_{|\alpha| \leq n}$ is a vector of unknown coefficients to be solved by using the reproducing conditions 


$$
\sum_{I=1}^{n} \Psi_{I}(\boldsymbol{x}) \boldsymbol{x}_{I}^{\alpha}=\boldsymbol{x}^{\alpha} \quad \alpha \leq n
$$

Imposing the reproducing conditions of (3) gives the discrete RK approximation

$$
\boldsymbol{u}^{h}(\boldsymbol{x})=\sum_{I} \boldsymbol{H}^{T}(\mathbf{0}) \boldsymbol{M}^{-1}(\boldsymbol{x}) \boldsymbol{H}\left(\boldsymbol{x}-\boldsymbol{x}_{I}\right) \Phi_{a}\left(\boldsymbol{x}-\boldsymbol{x}_{I}\right) \boldsymbol{d}_{I}
$$

where $\boldsymbol{M}(\boldsymbol{x})$ is a moment matrix defined as

$$
\boldsymbol{M}(\boldsymbol{x})=\sum_{I} \boldsymbol{H}\left(\boldsymbol{x}-\boldsymbol{x}_{I}\right) \boldsymbol{H}^{T}\left(\boldsymbol{x}-\boldsymbol{x}_{I}\right) \Phi_{a}\left(\boldsymbol{x}-\boldsymbol{x}_{I}\right) .
$$

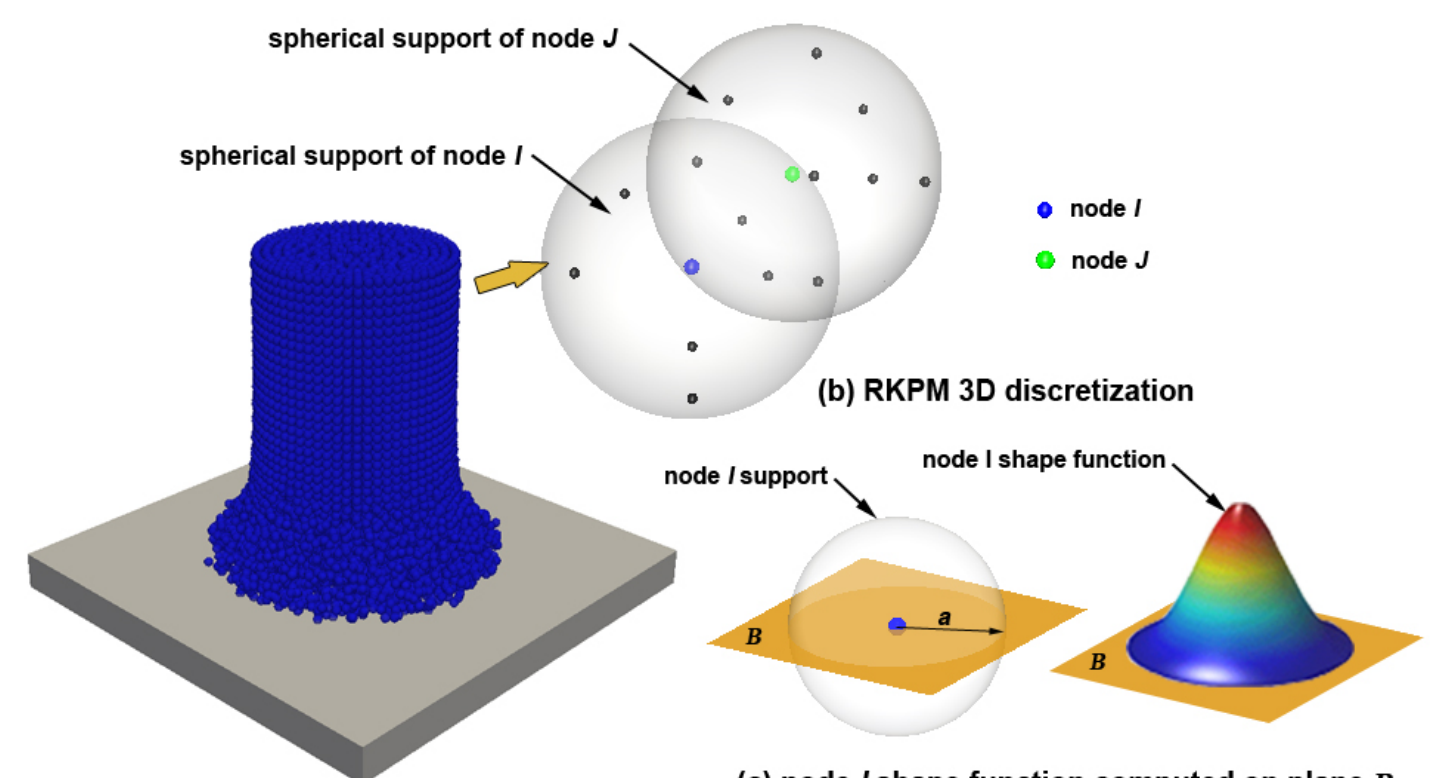

(a) RKPM model, Taylor bar impact

(c) node I shape function computed on plane $B$

Figure 1. RK discretization of Taylor bar impact.

\section{RANKINE-HUGONIOT JUMP CONDITION AND ENTROPY CONSTRAINT}

The Rankine-Hugoniot jump condition describes the relationship between the relative flux/solution jump across a shock and the shock-front velocity. For a numerical method to accurately capture the shock velocity, it must satisfy the Rankine-Hugoniot jump condition. Consider the Cauchy problem

$$
\begin{gathered}
u(x, t){ }_{, t}+f(u),_{x}=0 \\
u(x, 0)=\vartheta(x)
\end{gathered}
$$

where $u$ is the unknown field variable, $u(x, t),_{t}$ is the time derivative of $u$, and $f(u)$ is the flux function. Conservation equations in the form of (6) are used to describe inviscid flow, which can 
lead to shock formation even in the presence of initially smooth data. The weak form of Equation (6) integrated over an arbitrary space-time domain, $\Omega$, (reference Figure 2) is

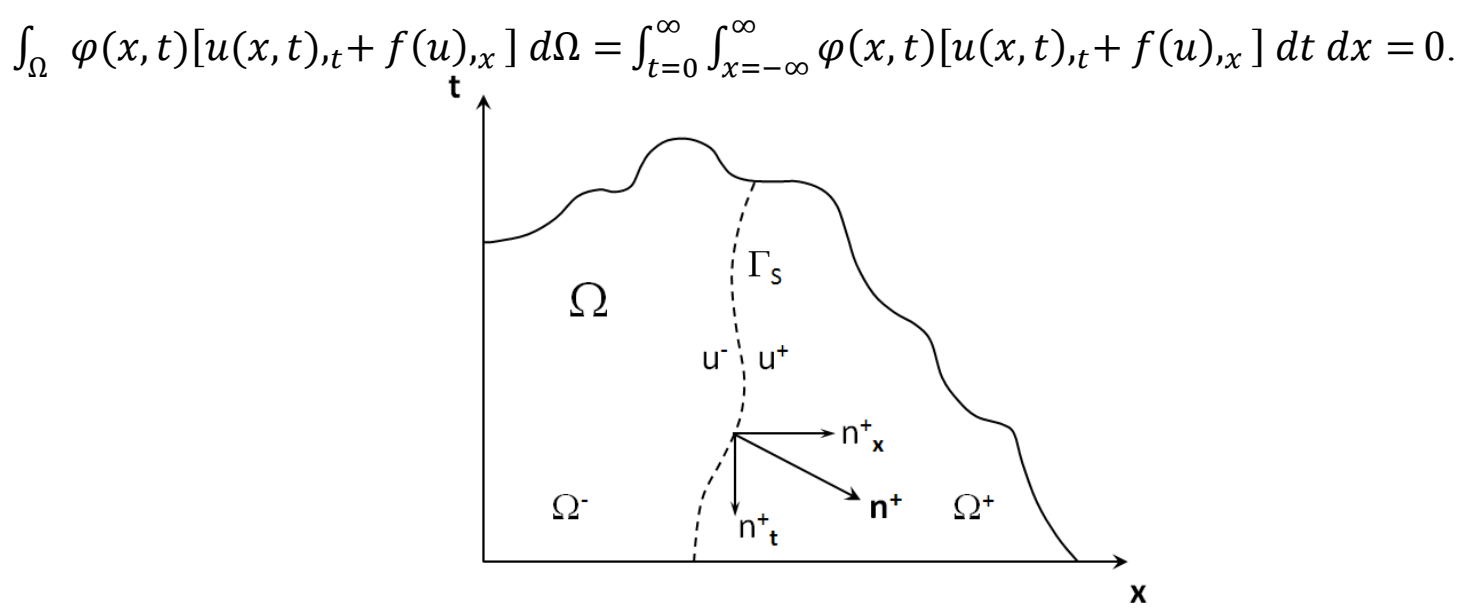

Figure 2. Arbitrary space-time domain containing discontinuity.

Using integration by parts and properties of the compact test function, $\varphi(\infty, t)=\varphi(x, \infty)=0$ and $\varphi(x, 0)=0$, Equation 8 is transformed to

$$
\int_{t=0}^{\infty} \int_{x=-\infty}^{\infty}\left[\varphi(x, t){ }_{t} u(x, t)+\varphi(x, t){ }_{x} f(u)\right] d t d x=-\int_{x=-\infty}^{\infty} \varphi(x, 0) \vartheta(x) d x=0 .
$$

In the presence of a shock, a discontinuity forms in $\Omega$ along the boundary $\Gamma_{\mathrm{s}}$ such that the discontinuity divides the space-time domain into two smooth space-time sub-domains, $\Omega^{-}$and $\Omega^{+}$. Normal to the discontinuity is $\boldsymbol{n}$, and the solutions to the left and right of the discontinuities are $u^{-}$and $u^{+}$, respectively. Considering the presence of the discontinuity, Equation (9) becomes

$$
\int_{\Omega^{-}}\left[\varphi,{ }_{t} u+\varphi,_{x} f(u)\right] d \Omega+\int_{\Omega^{+}}\left[\varphi,{ }_{t} u+\varphi,_{x} f(u)\right] d \Omega=0
$$

Using integration by parts and the divergence theorem in conjunction with the weak form expression of Equation (8) applied to the smooth sub-domains, Equation (10) becomes

$$
\int_{\Gamma_{\mathrm{s}}} \varphi\left[u^{-} n_{t}^{-}+f\left(u^{-}\right) n_{x}^{-}\right] d \Gamma=-\int_{\Gamma_{\mathrm{s}}} \varphi\left[u^{+} n_{t}^{+}+f\left(u^{+}\right) n_{x}^{+}\right] d \Gamma
$$

Due to arbitrariness of the test function, $\varphi$, and considering that $\boldsymbol{n}^{-}=-\boldsymbol{n}^{+}$, Equation (11) implies

$$
u^{-} n_{t}+f\left(u^{-}\right) n_{x}=u^{+} n_{t}+f\left(u^{+}\right) n_{x}
$$

The inverse of the slope of $\Gamma_{\mathrm{s}}$ defines the shock velocity, $v_{s}$, and is related to $\boldsymbol{n}$ by $v_{s}=d x / d t=-n_{t} / n_{x}$. Therefore, Equation (12) gives 


$$
v_{s}=\frac{f\left(u^{-}\right)-f\left(u^{+}\right)}{u^{-}-u^{+}}
$$

which is the Rankine-Hugoniot jump equation. This shows that the essential physics described by the Rankine-Hugoniot equation is embedded in the weak formulation.

The change in entropy, $S$, is also a key component of the shock-front physics. According to the second law of thermodynamics, for irreversible and adiabatic processes (such as a shock), entropy must increase across the shock front. It has been shown that this constraint is satisfied only when compressive discontinuities are present, e.g., [4]. Accordingly, this entropy constraint dictates the nature of the physically correct solution at a jump: compressive discontinuities propagate as a shock, and expansion discontinuities immediately degenerate into a rarefaction.

Unlike the case of the Rankine-Hugoniot jump condition, the weak formulation does not inherently guarantee satisfaction of the entropy constraint. Therefore, numerical methods for shocks are often enriched so that the entropy production criterion is satisfied. A so-called entropy condition, such as the Lax [5] shock condition

$$
\begin{gathered}
\lambda\left(u^{-}\right)>v_{s}>\lambda\left(u^{+}\right) \\
\lambda(u)=f^{\prime}(u)=\text { characteristic speed }
\end{gathered}
$$

or an entropy inequality [1],

$$
\begin{gathered}
\eta(u)_{t}+\Psi(u)_{\mathrm{x}} \leq 0 \\
\eta(u)=\text { entropy function, } \Psi(u)=\text { entropy flux }
\end{gathered}
$$

can be introduced to enforce the entropy-based, physically correct solution. Another approach to guarantee entropy satisfaction is use of an approximate Riemann solver (such as in the Godunov method), which solves a local Riemann problem to compute wave propagation. The approximate Riemann solver provides the additional advantage of limiting solution oscillation due to Gibbs phenomenon. In this work the approximate Riemann solver is used to enrich the RKPM formulation. As will be discussed in Section 5, the RKPM solution is locally corrected at the shock front using the flux jump computed from a local Riemann problem. This technique introduces the analytical shock solution at the jump so that the entropy production constraint is inherently enforced.

\section{SHOCK DETECTION BY SPECTRAL DECOMPOSITION}

The RK approximation possesses a unique spectral decomposition feature analogous to frequency filtering in wavelet filter analysis [6,7]. A wavelet filter is constructed from the convolution of an input signal, $g(x)$, and filter, $f(x)$, as 


$$
g^{*}(x)=g(x) * f(x)=\int_{-\infty}^{\infty} g(\tau) f(x-\tau) d \tau
$$

where $g^{*}(x)$ is the low-pass filtered output, and "*" is a convolution operator. The essential ingredient of the wavelet filter is the filter function, which is the basis of a nested subspace, $V_{m}$, where the subspaces, $V_{-\infty}, \cdots, V_{m-1}, V_{m}, V_{m+1}, \cdots, V_{\infty}=L^{2}(\mathbb{R})$, define a complete spectral decomposition of $L^{2}(\mathbb{R})$. Properties of the basis, $\Theta_{m n}(x)$, are defined by a window function, $\theta(x)$, such that

$$
f(x)=\Theta_{m n}(x)=2^{-m / 2} \theta\left(\frac{x}{2^{m}}-n\right)
$$

where $m$ and $n$ define the window dilation and translation, respectively. The RK approximation is also a convolution [6], where the approximation, $u^{h}(x)$, is

$$
u^{h}(x)=u(x) * \Phi_{a}^{n}(x)
$$

where $\Phi_{a}^{n}(x)$ is a so-called approximation kernel with basis order $n$ and compact support of size a.

The kernel function in Equation (20) behaves as a low-pass filter for which the filter limits are defined by the basis order and support size. The filter limit contracts with a decrease in basis order or an increase in support size. The RK approximation is the low-pass filtered output. You, Chen, and Lu (2003) defined another filter of the RK approximation as

$$
u^{f}(x)=u^{h}(x) * \Phi_{b}^{m}(x)
$$

where $\Phi_{b}^{m}(x)$ is a so-called filter kernel with basis order $m$ (not necessarily equal to $n$ ) and support size $b$ (not necessarily equal to $a$ ). In Equation (21), $u^{f}(x)$ is the low-pass component of the initial RK approximation. In discrete form, the filtered RK approximation is

$$
u^{f}(x)=u_{\text {low }}^{h}(x)=\sum_{I}\left[\sum_{J} \Psi_{I}^{n, a}\left(x_{J}\right) \Psi_{J}^{m, b}(x)\right] u_{I}
$$

The high-pass component of the RK approximation is trivially

$$
u_{\text {high }}^{h}(x)=u^{h}(x)-u_{\text {low }}^{h}(x)
$$

Numerical experiments using Equations (22) and (23) have been employed to study the basis and the support of the RK filter kernel to determine filter limits that accurately isolate the high-frequency signal in discontinuous approximations. Investigations with one- and twodimensional functions have shown that a single-order reduction in the filter kernel basis, $m=n-1$, and filter support 2 to 3 times larger than the approximation kernel support, $b=$ $2(a)$ or $b=3(a)$, typically provide sharp isolation of the discontinuity-induced high-frequency signal. An example is shown in Figure 3, where the RK approximation to a two-dimensional arc-shaped discontinuity was decomposed into high- and low-frequency components by using 
filter limits defined by $m=n-1$ and $b=3 a$. The high frequency signal is cleanly isolated to the discontinuity location.

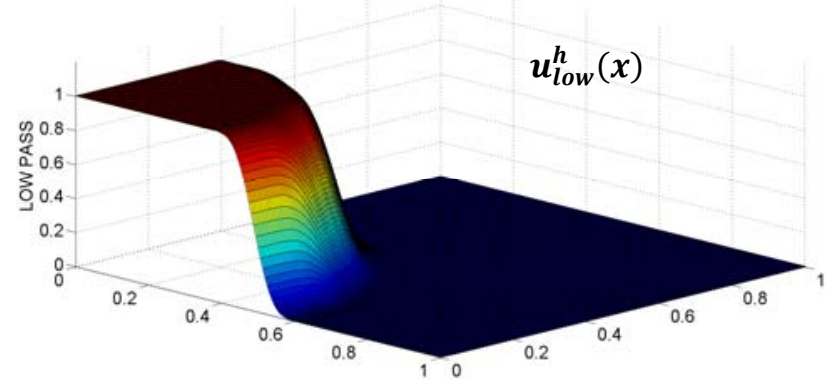

(a)

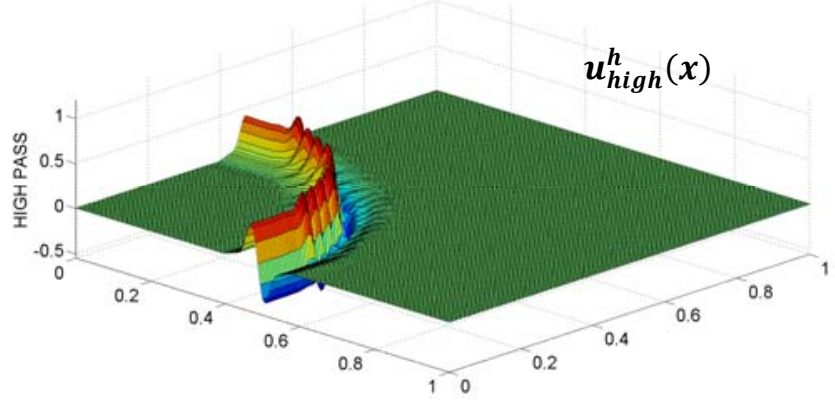

(b)

Figure 3. RK spectral decomposition of discontinuous function, (a) low-pass component and (b) high-pass component (using cubic B-spline kernel function).

It is well known that shocks exhibit a strong high-frequency signature at the jump location, as in Figure 3(b). Therefore, using the RK spectral decomposition feature, a detection algorithm can be constructed to detect and track transient shocks. With the shock location captured, techniques to enforce entropy satisfaction and control Gibbs phenomenon can be applied directly to the jump region.

In this work, a detection algorithm was developed by considering the high-frequency signature as a high-pass error. The relative density of the high-pass error can be used as an automatic shock indicator. The error measure is defined using the $L_{2}$ norm, so that the global high-pass error is

$$
e_{\text {global }}=\left\|u^{h}(x)-u_{\text {low }}^{h}(x)\right\|=\left\{\int_{\Omega}\left[u^{h}(x)-u_{\text {low }}^{h}(x)\right]^{2} d \Omega\right\}^{1 / 2}
$$

where $\Omega$ is the problem domain, as shown in Figure 4. The global error density is

$$
\bar{e}_{\text {global }}=\frac{e_{\text {global }}}{\int_{\Omega} d \Omega}
$$

The error and error density over a local subdomain, $\Omega_{L}$ (Figure 4), are similarly defined. Using the global and local error densities, the relative error density is

$$
e_{\text {rel }}=\frac{\bar{e}_{\text {local }}}{\bar{e}_{\text {global }}}
$$

where $e_{r e l}$ is a measure of the relative local error. To avoid false detection in smooth solutions, limiting criteria for the relative local error must be defined. From numerical experiments for one-dimensional problems, $e_{\text {rel }}$ of $75 \sim 125$ has been used as an indicator for strong shocks. 


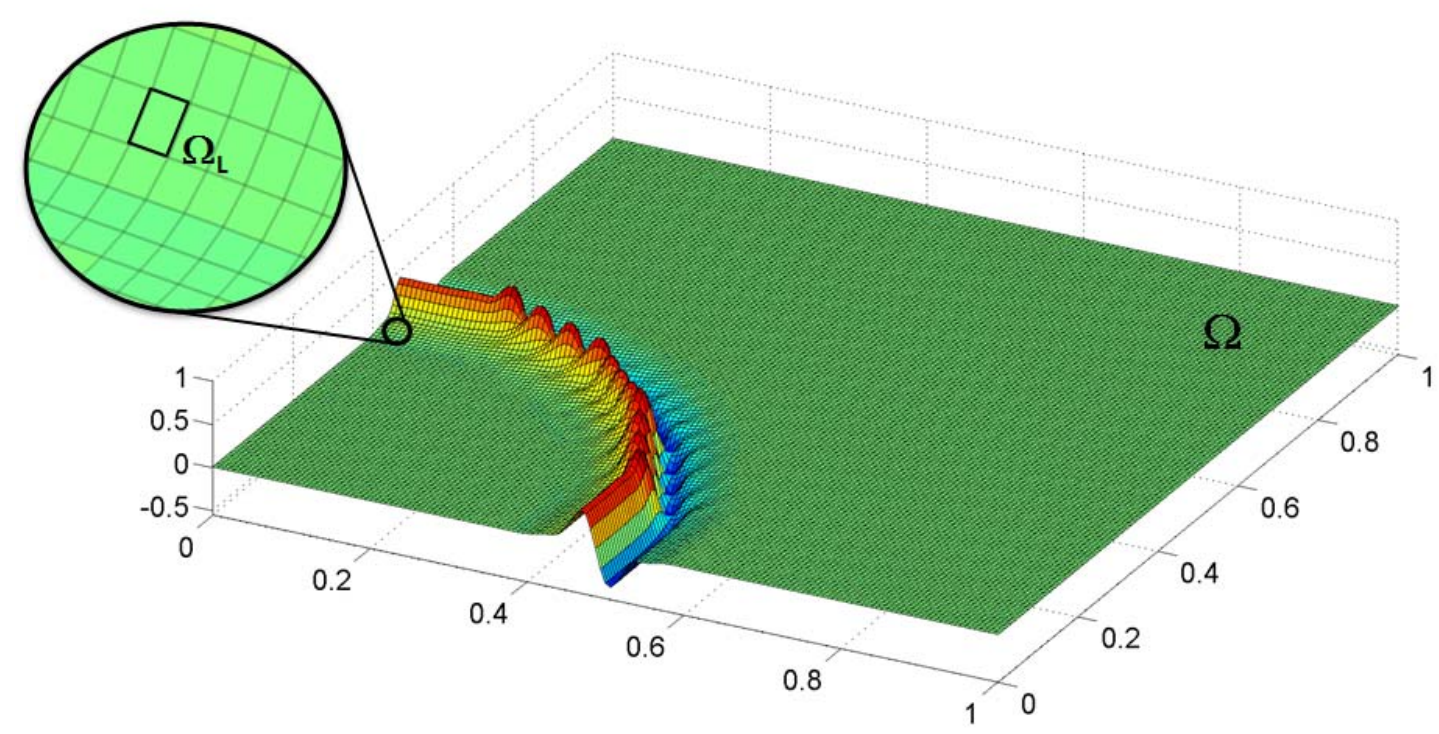

Figure 4. High-pass error analysis, global $(\Omega)$ and local $\left(\Omega_{\mathrm{L}}\right)$ domains.

\section{RKPM SHOCK MODELING TECHNIQUE}

The weak formulation does not necessarily guarantee satisfaction of the entropy production constraint. Further, higher-order methods are subject to oscillatory error at the shock front due to Gibbs phenomenon. Therefore, shock modeling techniques are required to enrich higher-order methods so that the key physics are satisfied and oscillatory error is minimized.

In this work, a flux-corrected velocity technique was developed for non-linear hyperbolic problems. The technique behaves as a local correction of the RKPM-computed velocity near shock-induced discontinuities. In the shock-front region, a local Riemann problem is constructed to compute the flux jump across the discontinuity. The corrected local velocities are computed in accordance with Godunov's scheme and are used in the temporal evolution equation to compute the corrected RKPM shock solution. A key feature of this technique is construction of the local Riemann problem in the shock region. The Riemann problem essentially introduces the analytical shock solution following characteristic projections at the jump. As a result, the physically correct solution (shock or rarefaction) is naturally captured, and the entropy constraint is inherently enforced. The other key feature of this technique is the introduction of the local Godunov solution to the RKPM formulation. The Godunov solution is first-order accurate and therefore behaves as a dissipative oscillation limiter at the jump. To maintain higher-order accuracy in smooth regions, the spectral decomposition shock detection algorithm is used to limit corrections to the jump location.

To construct the local Riemann problem (see Figure 5), the RKPM nodal solution near the discontinuity is projected in position-time space ( $x-t$ space) following characteristic lines, and the jump is determined to be shock forming if $u_{j}^{n}>u_{j+1}^{n}$ or rarefaction forming if $u_{j}^{n}<u_{j+1}^{n}$. 
The solution at the jump interface, $x_{j+1 / 2}$, is analytically determined from the Riemann problem by defining a local coordinate origin, $x_{j+1 / 2}=0$, and evaluating for the shock condition

$$
u_{j+1 / 2}= \begin{cases}u_{j} & \xi>0 \\ u_{j+1} & \xi<0\end{cases}
$$

or for the rarefaction condition

$$
u_{j+1 / 2}=\left\{\begin{array}{cc}
0 & u_{j}<0<u_{j+1} \\
u_{j} & \xi>0 \text { and } u_{j+1}>u_{j}>0 \\
u_{j+1} & \xi<0 \text { and } u_{j}<u_{j+1}<0
\end{array}\right.
$$

where $\xi$ is the analytical shock velocity defined for the governing equation. Given the projected solution at the jump interface, the interface flux, $f\left(u_{j+1 / 2}^{n}\right)$, is determined.

The evolution equation for Godunov's scheme is

$$
u_{j}^{n+1}=u_{j}^{n}-\Delta t\left(\frac{f_{j+1 / 2}^{n}-f_{j-1 / 2}^{n}}{\Delta x}\right) \equiv u_{j}^{n}-\Delta t(\mathcal{F})
$$

where $\mathcal{F}$ is the flux-based velocity that projects the solution forward in time. For the RKPM temporal integration scheme, consider the simple generalized trapezoidal rule

$$
u_{j}^{n+1}=u_{j}^{n}+\Delta t(1-\alpha) \dot{u}_{j}^{n}+\alpha \Delta t \dot{u}_{j}^{n+1}
$$

where $\alpha$ controls solution accuracy. Re-writing Equation (30) in a form similar to (29) gives

$$
u_{j}^{n+1}=u_{j}^{n}+\Delta t\left[(1-\alpha) \dot{u}_{j}^{n}+\alpha \dot{u}_{j}^{n+1}\right]=u_{j}^{n}+\Delta t(\mathbb{F}) .
$$

Therefore, to introduce the Godunov scheme to the RKPM formulation, the temporal integration scheme in the shock region is modified so that the solution is projected in time with the flux-based velocity (i.e., $\mathbb{F}=-\mathcal{F}$ ). Because the solution of the Riemann problem is embedded in the flux-based velocity, the resulting RKPM scheme is entropy satisfying and oscillation limited. 

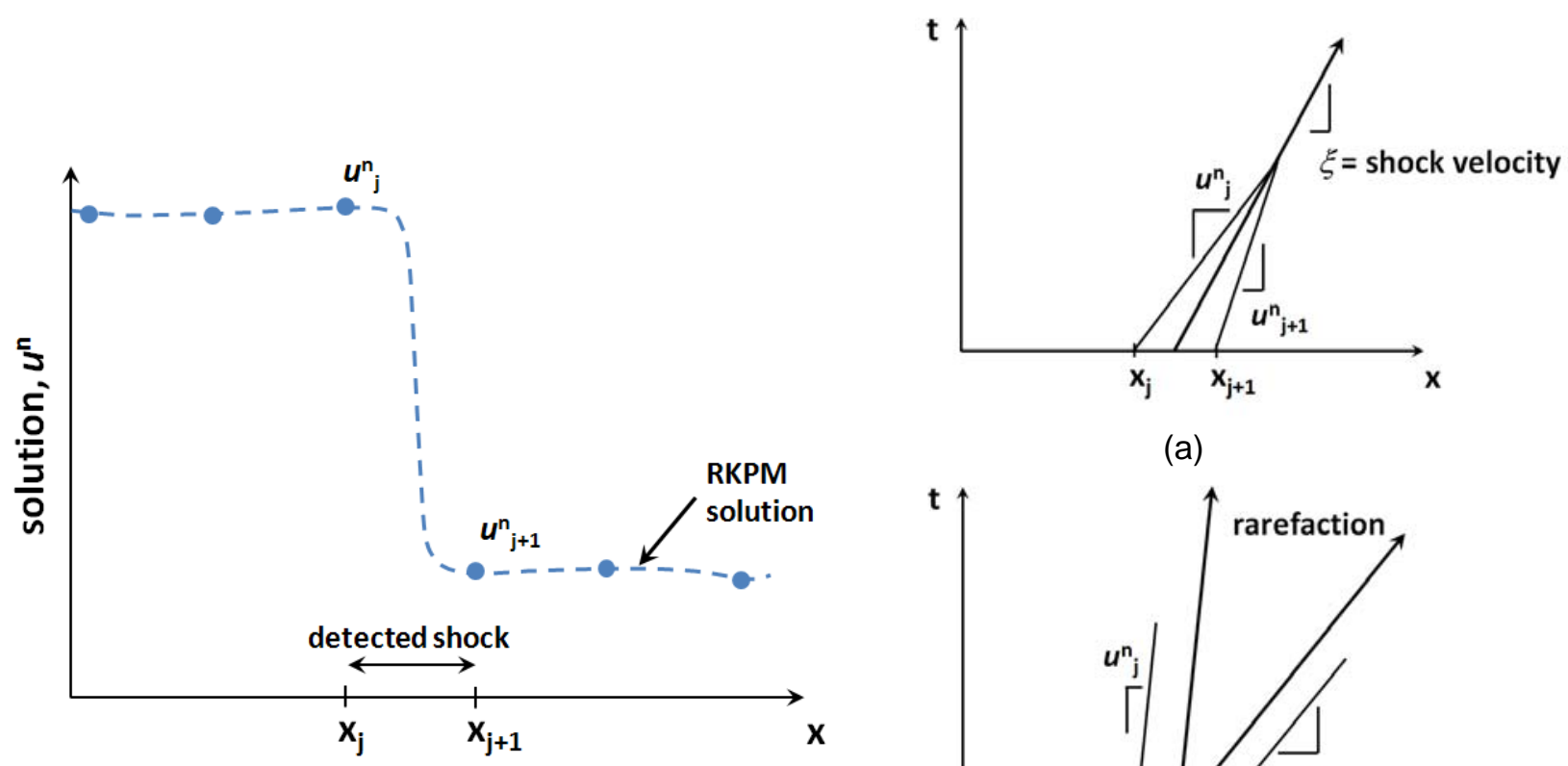

(a)

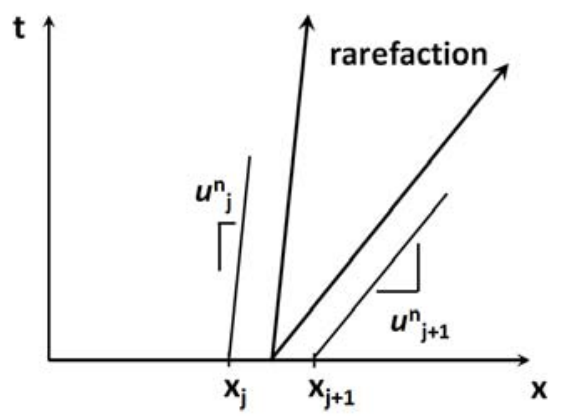

(b)

Figure 5. RKPM near-discontinuous solution at time $t^{n}$ and local Riemann problem, (a) shock forming, $u_{j}>u_{j+1}$, or (b) rarefaction forming, $u_{j}<u_{j+1}$.

The computational procedure for this flux-corrected velocity technique is

1. Compute the higher-order accurate RKPM solution over the full computational domain at time $t^{n+1}$

2. Using the shock detection algorithm, detect a shock formation near $x_{j}$ and define a thin region near the shock that encompasses $x_{j-\gamma}, \cdots, x_{j}, \cdots, x_{j+\gamma}$;

3. In the shock region,

a. compute the local Riemann solution using Equations (27) and (28),

b. compute the flux-corrected velocity, $\mathcal{F}$, and

c. compute the corrected solution using $\mathbb{F}=-\mathcal{F}$ in Equation (31);

4. Replace the initial RKPM solution in the shock region with the flux-corrected solution and advance to the next time step.

Using this technique, the higher-order-accurate RKPM solution is maintained over the smooth solution regions, and the first-order accurate flux-corrected solution is applied only in a thin region at the shock. 


\section{NUMERICAL EXAMPLE}

The solution of the inviscid Burgers equation was solved to show the accuracy provided by the flux-corrected velocity RKPM formulation. Burgers equation is a non-linear hyperbolic partial differential equation (PDE) commonly used as a model problem for shocks. Smooth initial conditions defined on a domain $x \in[0,4]$ were prescribed by a cubic B-spline function. The solution of this PDE forms a shock at approximately $\mathrm{t}=0.45$. A rarefaction also forms behind the shock, so that the model problem investigates performance for both shock and rarefaction conditions. The RKPM solution was computed using a linear basis and normalized support ( $a / h$ where $a$ is compact support size and $h$ is nodal spacing) equal to 1 . The spatial domain was discretized with a uniform nodal spacing of 0.0125 . Central difference time integration was used with the time step defined by $\Delta t / \Delta x=0.25$. This formulation is secondorder accurate (in the $L_{2}$ error norm) in space and time. For the shock detection algorithm, 125 was used for the relative error density indicator, $e_{r e l}$; and the filter kernel basis and normalized support were $m=0$ and $b=2 a$, respectively.

In Figure 6, the RKPM solution is compared to the analytical before the shock forms and a fine-scale Lax-Friedrichs (LF) solution $(\Delta x=0.002)$ after the shock forms. Solutions are compared at $\mathrm{t}=0.375, \mathrm{t}=1.25$, and $\mathrm{t}=5.5$ in Figures 6(a), 6(b), and 6(c), respectively. At $\mathrm{t}=0.375$ the shock has not fully formed. Accordingly, the RKPM solution was not corrected, and secondorder accuracy was maintained over the full domain. At $\mathrm{t}=1.25$ and $\mathrm{t}=5.5$, the detection algorithm automatically identified a thin region containing the shock. The flux-corrected velocity was applied in this region, and therefore accuracy was reduced to first order at the front. However, since the detection algorithm isolated the correction to the front, higher-order accuracy was maintained elsewhere. Although the solution in the shock region was reduced to first order, the RKPM solution did not exhibit the same dissipative error as the first-order accurate LaxFriedrichs solution. The RKPM and Lax-Friedrichs shock velocities were in very close agreement as a result of the Rankine-Hugoniot condition's embedment in the weak formulation. The slight difference in shock velocities was the result of integration error in the weak form. In Figure $6(\mathrm{~d})$, the solution at $\mathrm{t}=1.25$ without the flux-corrected velocity is shown for comparison with Figure 6(b) using flux-corrected RKPM. The solution exhibited large oscillatory error at the discontinuity due to Gibbs phenomenon. 


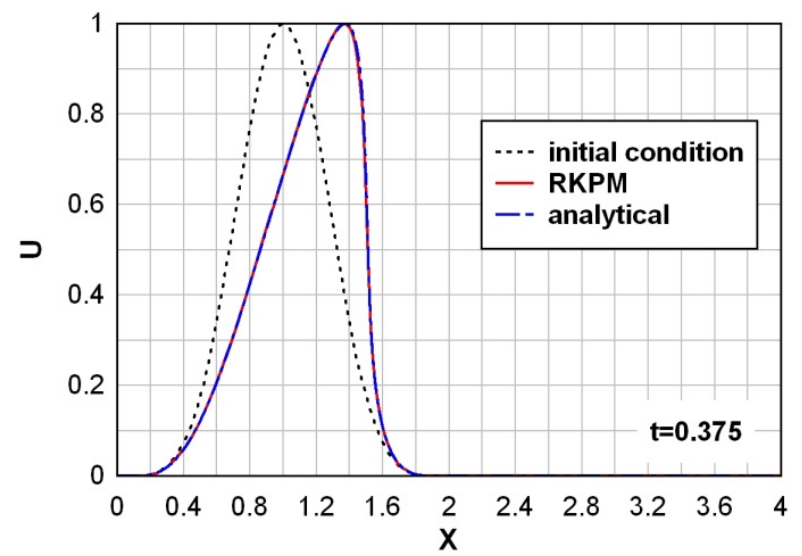

(a)

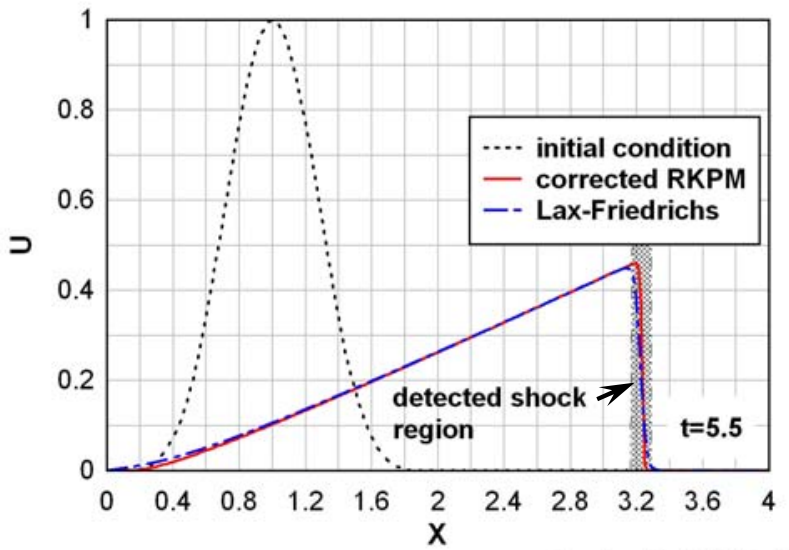

(c)

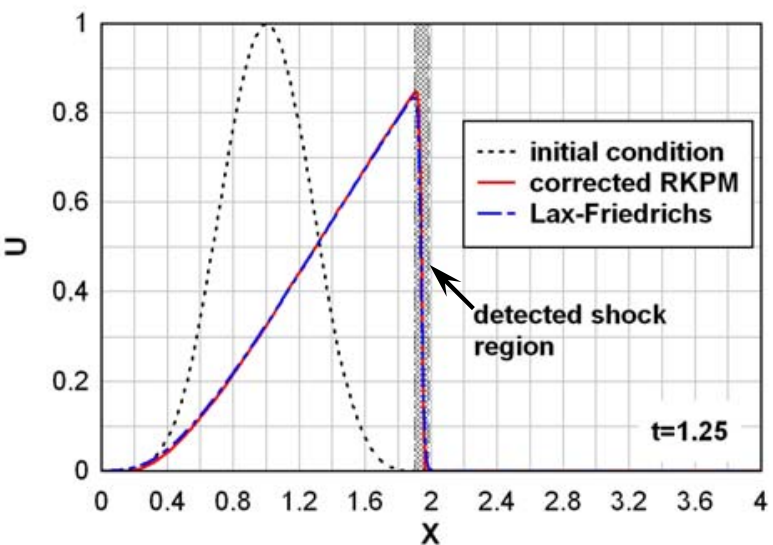

(b)

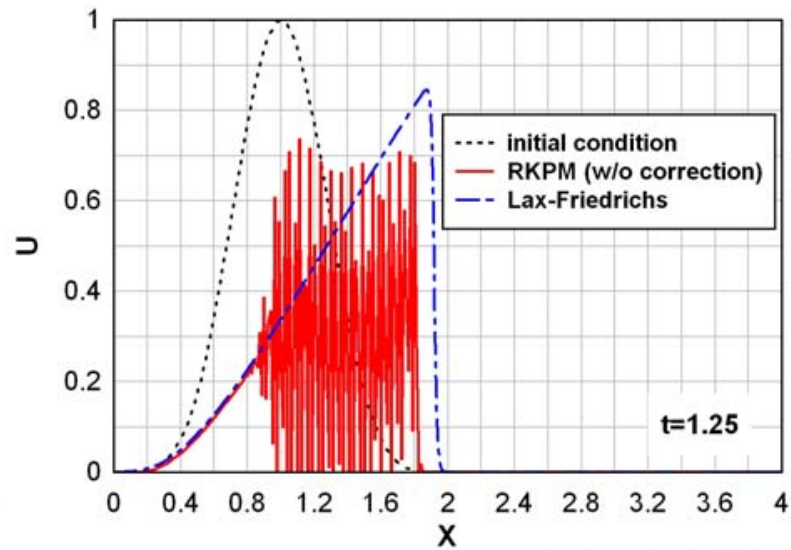

(d)

Figure 6. Solution to the inviscid Burgers equation, (a) RKPM and analytical solution prior to shock, $\mathrm{t}=0.375$, (b) RKPM and LF solution, $\mathrm{t}=1.25$, (c) RKPM and LF solution, $\mathrm{t}=5.5$, and (d) oscillatory solution without flux correction.

\section{SUMMARY AND CONCLUSIONS}

To accurately model shock effects, numerical methods must contain the appropriate shock physics and minimize oscillatory error generated by Gibbs phenomenon at the jump. RKPM is derived from the weak formulation and naturally satisfies the Rankine-Hugoniot jump condition. However, satisfaction of entropy production in accordance with the second law of thermodynamics is not guaranteed. Enrichment of the RKPM formulation has been developed using a flux-based velocity correction at the shock front. The correction is derived from the Riemann solution to a locally defined Cauchy problem and therefore enforces the physically correct solution in accordance with the entropy constraint. Since the correction is built into the formulation following a Godunov-type scheme, the correction also behaves as an oscillation limiter to control Gibbs phenomenon effects. The spectral decomposition property inherent to RKPM is used to construct a detection algorithm so that the correction is applied only in a thin 
region at the front. The correction only reduces solution accuracy to first order locally, and higher order accuracy remains away from the short front. In this way, the technique is similar to other oscillator-limiting schemes. The solution of the inviscid Burgers equation was computed to show the technique's effectiveness. Using a second-order accurate space/time formulation, the flux-uncorrected RKPM solution is shown to be highly oscillatory (as would be the case with other methods, such as the finite element method). The RKPM with flux correction eliminated oscillation and reduced the dissipative error as compared to a fine-scale Lax-Friedrichs solution. This technique has been investigated in the one-dimensional case. Further investigation is required for the extension to higher dimensions and application to the equation of motion for solid dynamics.

\section{Acknowledgements}

Permission to publish was granted by Director, Geotechnical and Structures Laboratory, U.S. Army Engineer Research and Development Center.

\section{REFERENCES}

[1] R.J. Leveque, Numerical Methods for Conservation Laws, Birkhauser, Basel, 1992.

[2] J.S. Chen, C. Pan, C.T. Wu, and W.K. Liu, Reproducing kernel particle methods for large deformation analysis of nonlinear structures, Computer Methods in Applied Mechanics and Engineering, 139, 49-74, 1996.

[3] W.K. Liu, S. Jun, and Y.F. Zhang, Reproducing kernel particle methods, International Journal for Numerical Methods in Fluids, 20, 1081-1106, 1995.

[4] A. Jeffrey and T. Tanuiti, Non-linear wave propagation: Applications to physics and magnetohydrodynamics," Mathematics in Science and Engineering, 9, ed. R. Bellman, Academic Press, New York, 1964.

[5] P. Lax, Hyperbolic systems of conservation equations, II, Communications in Pure and Applied Mathematics, 10, 537-566, 1957.

[6] W.K. Liu and Y. Chen, Wavelet and multiple scale reproducing kernel methods, International Journal for Numerical Methods in Fluids, 21, 901-931, 1995.

[7] Y. You, J.S. Chen, and H. Lu, Filters, reproducing kernel, and adaptive meshfree method, Computational Mechanics, 31, 316-326, 2003.

[8] P. C. Guan, S. W. Chi, J. S. Chen, T. R. Slawson, M. J. Roth, Semi-Lagrangian Reproducing Kernel Particle Method for Fragment-Impact Problems, International Journal of Impact Engineering, 38, 1033-1047, 2011. 